\title{
POLYNOMIAL INVARIANTS OF GRAPHS
}

\author{
SEIYA NEGAMI
}

\begin{abstract}
We define two polynomials $f(G)$ and $f^{*}(G)$ for a graph $G$ by a recursive formula with respect to deformation of graphs. Analyzing their various properties, we shall discuss when two graphs have the same polynomials.
\end{abstract}

Introduction. Our graphs are finite, undirected ones with or without self-loops and multiple edges. As elementary deformations of graphs, we have the deletion and the contraction of edges. Especially we define the contraction of an edge $e$ as an operation which deletes $e$ and identifies its ends to a single vertex. If an edge $e$ is a loop, its deletion and contraction are identical. We denote the resulting graph of deletion and contraction of an edge $e \in E(G)$ by $G-e$ and $G / e$, respectively.

We define a polynomial $f(G ; t, x, y)$ for a graph $G$ as follows. (We shall often simply write $f(G)$.)

(i) $f\left(\overline{K_{n}}\right)=t^{n}(n \geq 1)$.

(ii) $f(G)=x f(G / e)+y f(G-e)(e \in E(G))$.

The graph $\overline{K_{n}}$ is the complement of the complete graph $K_{n}$ over $n$ vertices, that is, it consists of only $n$ isolated vertices. The repetition of deleting and contracting edges transforms finally into $\overline{K_{n}}$, so $f(G)$ can be determined by the above two formulas. The well-definedness of $f(G)$ will be shown in Theorem 1.1. It is convenient to set $f(\varnothing)=1$ for the null graph $\varnothing$ which has no vertex and no edge.

If there is an isomorphism $\sigma: G \rightarrow H$ between two graphs $G$ and $H$, then $f(G)$ and $f(H)$ can be calculated in parallel via $\sigma$ and $f(G)=f(H)$. So this polynomial $f(G)$ is an isomorphism invariant.

As is shown in $\S 2, f(G)$ includes various information about a graph $G$, namely the numbers of subgraphs, cutsets, vertex-colorings, flows and so on. So $f(G)$ seems to be a very useful invariant for the isomorphism problem of graphs. However there are many graphs whose isomorphism types cannot be distinguished by $f(G)$. For example, all trees with the same number of edges, say $q$, have the same polynomial $t(x+t y)^{q}$.

Our arguments in the last half of this paper will show that $f(G)$ is a 2-isomorphism invariant rather than an isomorphism invariant. The definition of 2 -isomorphism can be found in [5] and will be given in $\S 5$. Roughly speaking, two graphs are said to be 2-isomorphic if they can be transformed into each other by certain kinds of modifications at 1- or 2-vertex cuts. (Our 2-isomorphism is slightly different from the ordinary one. We do not regard two graphs to be 2-isomorphic when one is obtained from the other by breaking it at a cut vertex. Under our definition, 2-isomorphic graphs have the same number of components.) In particular, when a

Received by the editors February 12, 1986.

1980 Mathematics Subject Classification (1985 Revision). Primary 05C99.

Key words and phrases. Graphs, polynomials, 2-isomorphism. 
graph $G$ is planar, such modifications generate all the duals of $G$. That is, any two duals of one planar graph are 2-isomorphic.

In $\S 3$, we define another polynomial $f^{*}(G)$ for a graph $G$ in a similar way but slightly complicated and call it the dual polynomial of $G$. For a beautiful duality holds when $G$ is planar;

$$
f^{*}(G)=f\left(G^{*}\right) .
$$

Any dual $G^{*}$ of a planar graph $G$ is associated with an embedding of $G$ on a sphere. Conversely, the variation of spherical embeddings of $G$ induces many different duals. The above formula however implies that all duals of $G$ have the same polynomial. For example, all trees with $q$ edges are duals of a single vertex with $q$ self-loops. That is why they have the same polynomial.

In order to analyze these phenomena, we shall consider a resolution of a graph $G$, defined in $\S 1$, which is naturally associated with a process for calculation of $f(G)$ and establish a beautiful formula (Theorem 4.2) in $\S 4$. Assume that a graph $G$ splits into two subgraphs $K$ and $H$ which meet in only $n$ common vertices, $U=V(K) \cap V(H)$. Let $\Gamma(U)$ denote the partition lattice over $U$ and let $\gamma$ be any member of $\Gamma(U)$ which partitions $U$ into $k$ subsets $U_{1}, \ldots, U_{k}$. We denote by $K / \gamma$ (or $H / \gamma$ ) the graph obtained from $K$ (or $H$ ) by identifying all vertices in each $U_{i}$ to one vertex. (If each $U_{i}$ is a singleton, $K / \gamma$ coincides with $K$ itself.) We shall show that there are rational functions $B_{n}(\gamma, \delta ; t)$ with only one variable $t$ for any pair $(\gamma, \delta)$ of members of $\Gamma(U)$ such that

$$
f(G)=\sum_{(\gamma, \delta) \in \Gamma(U) \times \Gamma(U)} f(K / \gamma) B_{n}(\gamma, \delta ; t) f(H / \delta) .
$$

It is important here that $B_{n}(\gamma, \delta ; t)$ does not depend on $K$ and $H$. The above formula, called the splitting formula, splits $f(G)$ into three parts which can be calculated from only $K, H$ and the size of $U$ independently. (The Tutte polynomial, one of most famous polynomial invariants of graphs, does not split so nicely as our $f(G)$. If one expands it into the same type expression, then $B_{n}(\gamma, \delta ; t)$ will contain a factor depending on the number of components of $K / \gamma$ and $H / \delta$.)

It is routine but hard to give the explicit expressions of $B_{n}(\gamma, \delta ; t)$ 's when $n$ is a large number. In particular, if $K$ and $H$ are disjoint $(n=0)$ or one-point join $(n=1)$, we have $f(G)=f(K) f(H)$ or $f(G)=f(K) f(H) / t$, respectively. (The former is known as one of axioms of $V$-functions defined by Tutte [3].) We shall show our splitting formulas with explicit styles for $n=1,2,3$. When $n \geq 4$, they will be too long and complicated.

In $\S 5$, we shall discuss when two graphs have the same polynomial, as an application of our splitting formula, and especially show that any two 2-isomorphic graphs have the same polynomial. Our arguments there will suggest how we construct a pair of graphs with the same polynomial but not 2-isomorphic.

1. Resolutions of graphs. Our goal in this section is to know what the polynomial $f(G)$ is. Our discussion here will be a basis for later sections. We denote the numbers of vertices, edges and components of a graph $G$ by $p(G), q(G)$ and $\omega(G)$, respectively, and we shall often write simply $p, q$ and $\omega$ instead of them throughout. We set $\beta(G)=q(G)-p(G)+\omega(G)$ and call $\beta(G)$ the Betti number of $G$. This is equal to the number of edges not belonging to a spanning forest. 
First of all, we have to prove the well-definedness of $f(G)$, showing that $f(G)$ does not depend on the choice of an edge $e$ in definition.

THEOREM 1.1. The polynomial $f(G)$ is well defined.

ProOF. When $G$ has at most one edge, there is no ambiguity in the definition of $f(G)$, so $f(G)$ is uniquely evaluated. Suppose that $G$ has at least two edges, say $e, d \in E(G)$. Since the deletion and contraction of edges commute $((G / e)-d=$ $(G-d) / e$, etc. $)$, we have

$$
\begin{aligned}
& x f(G / e)+y f(G-e) \\
& \quad=x(x f((G / e) / d)+y f((G / e)-d))+y(x f((G-e) / d)+y f((G-e)-d)) \\
& \quad=x(x f((G / d) / e)+y f((G / d)-e))+y(x f((G-d) / e)+y f((G-d)-e)) \\
& \quad=x f(G / d)+y f(G-d) .
\end{aligned}
$$

This implies that the formula (ii) determines $f(G)$ uniquely.

Notice that the initialization $f\left(\overline{K_{n}}\right)=t^{n}$ is not essential in the above proof. So we can define other well-defined polynomials by setting the values of $\overline{K_{n}}$ arbitrarily. For example, define another polynomial $f^{\prime}(G)$ with $f^{\prime}\left(\overline{K_{n}}\right)=n$. Then we have

$$
f^{\prime}(G)=(\partial f(G) / \partial t)_{t=1} \text {. }
$$

Some of our arguments work for $f^{\prime}(G)$ in parallel, but the splitting formulas, Theorems 1.5 and 4.2, do not hold for it. We should take reasonable initial values for polynomials.

Now we shall consider binary trees naturally associated with the recursive processes for calculation of $f(G)$. We shall formulate them as families of deformed graphs with certain relations, as follows.

A string of letters $x$ and $y$, say $x x y x y$, is called a word in $x$ and $y$ and the total number of letters is called its length. Especially, we denote the empty word, that is, the unique word of length 0 by $\varnothing$. A set $W$ of words is said to be tree-like if the following (i) and (ii) hold:

(i) $\varnothing \in W$.

(ii) If $w x$ (or $w y) \in W$ for a word $w$, then $w, w y, w x \in W$.

Every tree-like set $W$ corresponds to a directed binary tree $T(W)$ with labels $x$ and $y$ on edges and is realized as the set of sequences of $x$ and $y$ obtained by reading off labels along paths from the unique source of $T(W)$. Each vertex of $T(W)$, except its source and sinks, is incident to only one incoming edge and exactly two outgoing edges labeled $x$ and $y$ each. Since any two vertices are joined by a unique path in a tree, we assign the word along each path in $T(W)$ to the terminus of the path. A word of $W$ corresponding to a sink of $T(W)$ is called an end of $W$ and we denote the set of ends by $\Omega(W)$;

$$
\Omega(W)=\{w \in W \mid w x, w y \notin W\} .
$$

Let $W$ be a tree-like set of words in $x$ and $y$ and let

$$
R(G ; W)=\{G[w] \mid w \in W\}
$$

be a family of graphs. We call $R(G ; W)$ a resolution of a graph $G$ if the following (i) and (ii) hold:

(i) $G(\varnothing)=G$. 


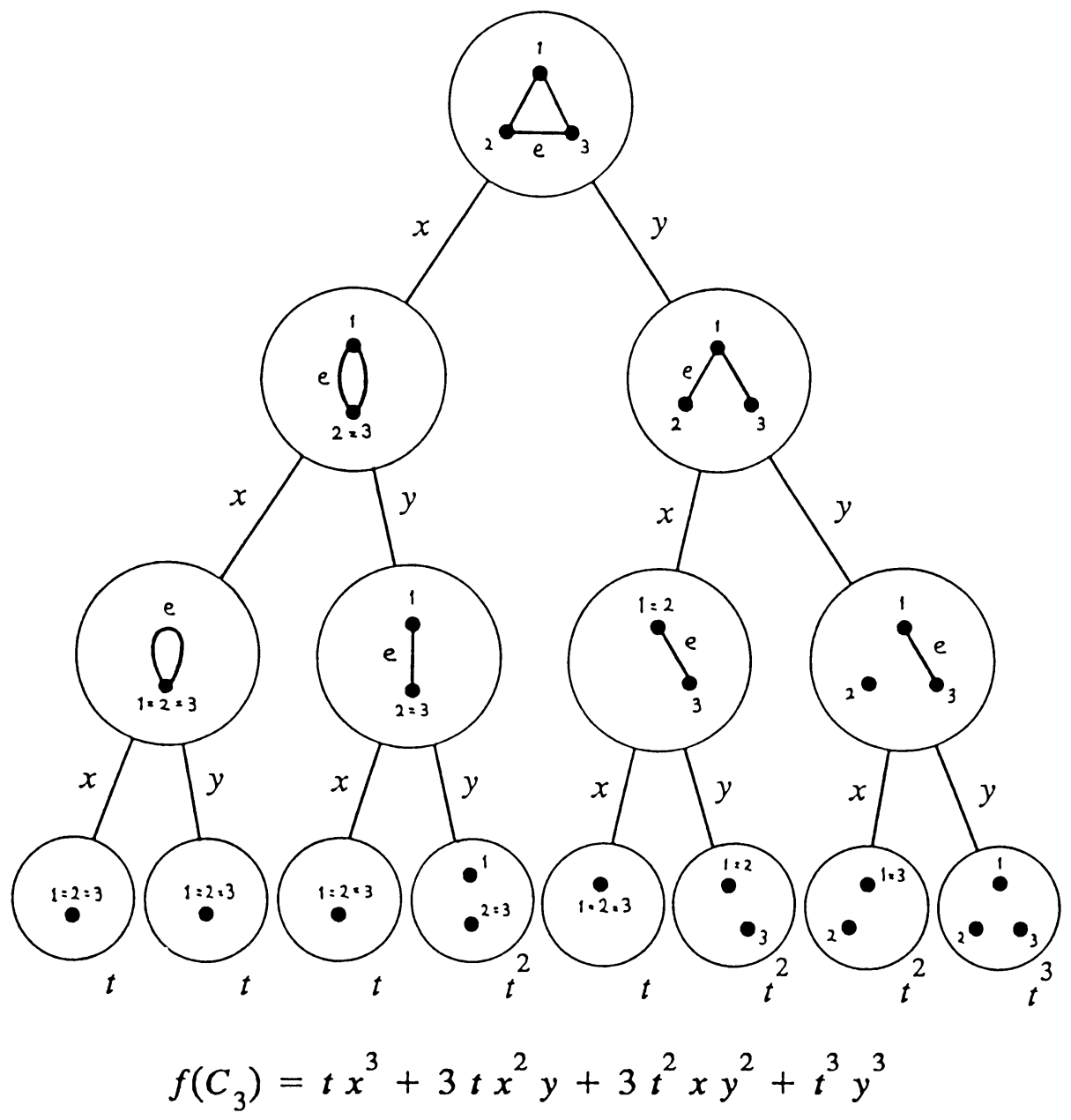

FigURE 1. A resolution of $C_{3}$.

(ii) When $w, w x, w y \in W$, then

$$
G(w x)=G(w) / e(w), \quad G(w y)=G(w)-e(w)
$$

for some edge $e(w)$ of $G(w)$.

Especially, when $W$ consists of all the words of length less than or equal to $n$, $R(G ; W)$ is called a resolution of depth $n$. Moreover if $n$ coincides with the number of edges of $G, R(G ; W)$ is a total resolution, and otherwise a partial resolution. Let $e_{1}, \ldots e_{q}$ be the edges of $G$ with labels $1, \ldots, q$. If $e(w)=e_{k}$ for every word $w$ of length $k$, then $R(G ; W)$ is said to be standard.

We consider a resolution $R(G ; W)$ intuitively as the binary tree $T(W)$ with each $G(w)$ assigned to the corresponding vertex, rather than a family of graphs. For example, we imagine a configuration like Figure 1 for a standard total resolution of the cycle $C_{3}$ of length 3 . Each branch has a weight $x$ or $y$ corresponding to contraction (left) or deletion (right), respectively. 
Let $[w]$ be $w$ with the order of occurrences of $x$ and $y$ neglected. For example, $[x x y x y x]=[x y y x x x]$ and they are identified with $x^{4} y^{2}$. Then we have the following formula:

THEOREM 1.2. For every resolution $R(G ; W)$ of a graph $G$,

$$
f(G)=\sum_{w \in \Omega(W)}[w] f(G(w)) .
$$

Let $w=l_{1} l_{2} \cdots l_{n}\left(l_{i} \in\{x, y\}\right)$ be a word of length $n$ in a tree-like set $W$ and let $w_{k}(k=1,2, \ldots, n)$ denote the subword $l_{1} l_{2} \cdots l_{k}$ of first $k$ letters in $w$. Then each graph $G(w)$ in a resolution $R(G ; W)$ is obtained from $G$ by contracting $X(w)=\left\{e\left(w_{k}\right): l_{k}=x\right\}$ and deleting $Y(w)=\left\{e\left(w_{k}\right): l_{k}=y\right\}$, so we write $G(w)=(G-Y(w)) / X(w)$.

Now let $R(G ; W)$ be a standard total resolution of a graph $G$. Each end word $w \in \Omega(W)$ induces a partition of $E(G)$ into the two disjoint sets $X(w)$ and $Y(w)$. The collection

$$
\{(X(w) ; Y(w)): w \in \Omega(W)\}
$$

contains all $2^{q}$ partitions of $E(G)$ into two parts and no duplication. Since $G(w)$ $(w \in \Omega(W))$ has no edge, $f(G(w))=t^{p(G(w))}$ for the number $p(G(w))$ of vertices of $G(w)$. We notice that if two vertices of $G$ are joined by a path of edges in $X(w)$ then they are identified to the same vertex in $G(w)$, and hence $p(G(w))=\omega(G-Y(w))$. Therefore, we have the following expansion of $f(G)$.

THEOREM 1.3. $f(G)=\sum_{Y \subset E(G)} t^{\omega(G-Y)} x^{q-|Y|} y^{|Y|}$.

This expansion of $f(G)$ does not depend on a resolution of $G$, so it also implies the well-definedness of $f(G)$.

The polynomial $f(G)$ expands generally into

$$
f(G)=\sum_{i=0}^{q} \sum_{j=1}^{p} b_{i j} t^{j} x^{q-i} y^{i}
$$

where $b_{i j}$ is a nonnegative integer. From Theorem 1.3, we can conclude what each coefficient $b_{i j}$ is.

THEOREM 1.4 (CUTSETS AND SPANNING SUBGRAPHS). (i) $b_{i j}=$ the number of subsets $Y$ of $i$ edges in $E(G)$ such that $G-Y$ has precisely $j$ components.

(ii) $b_{i j}=$ the number of spanning subgraph of $G$ with precisely $q-i$ edges and $j$ components.

If a graph $G$ is a union of two subgraphs $K$ and $H$ which meet in only a cut vertex of $G$, then $G$ is called a one-point join of $K$ and $H$ and is denoted by $K \cdot H$. The cutsets of $K$ and of $H$ are nearly independent in a disjoint union or a one-point join of $K$ and $H$. Thus, Theorems 1.3 and 1.4 imply immediately that $f(G)$ splits into the product of $f(K)$ and $f(H)$, as follows. The notation $K \cdot H$ is ambiguous but the ambiguity has no effect on the polynomial $f(G)$.

THEOREM 1.5. (i) If $K$ and $H$ are disjoint, then

$$
f(K \cup H)=f(K) f(H) .
$$


(ii) If $K$ and $H$ intersect in exactly one vertex, then

$$
f(K \cdot H)=f(K) f(H) / t .
$$

PROOF. Here we shall show it by induction on the number of edges. If both $K$ and $H$ consist of only isolated vertices, clearly (i) and (ii) hold. In case of one-point joins, we have

$$
\begin{aligned}
f(G) & =x f(G / e)+y f(G-e)=x f(K / e) f(H) / t+y f(K-e) f(H) / t \\
& =(x f(K / e)+y f(K-e)) f(H) / t=f(K) f(H) / t
\end{aligned}
$$

by the definition of $f(G)$ and the induction hypothesis. Eliminate "/ $t$ " in the above for (i).

2. What $f(G)$ tells us. By Theorem 1.4, the polynomial $f(G)$ gives us information about cutsets and spanning subgraphs of a graph $G$. More concretely, we can read off various properties of $G$ from suitable coefficients $b_{i j}$ of $f(G)$. We shall list them here.

It is easy to know the three most basic invariants $p(G), q(G)$ and $\omega(G)$, namely the numbers of vertices, edges and components. The sum of exponents of $x$ and $y$ in each term of $f(G)$ is equal to $q(G)$ constantly. If one goes along the most left side of a standard total resolution of a graph $G$, reading $x, x, x, \ldots$, a graph $G$ shrinks to points component-wise. Thus, $f(G)$ has a unique term which contains $x^{q}$ and it is $t^{\omega} x^{q}$. On the other hand, if one goes along the most right side, now reading $y, y, y, \ldots$, all edges of $G$ are deleted and there remain only vertices. So this path yields the unique term $t^{p} y^{q}$.

THEOREM 2.1 (EDGes, Vertices AND COMPONENTS). (i) The degrees of $f(G)$ in $x$ and in $y$ are equal to the number of edges of $G$.

(ii) $b_{0 \omega}=1$ and $b_{0 j}=0$ for any $j \neq \omega$.

(iii) $b_{q p}=1$ and $b_{q j}=0$ for any $j \neq p$.

COROllary 2.2 (EDges, VERTICES AND COMPONENTS). (i) $q(G)=$ $\log _{2} f(G ; 1,1,1)=\log _{2} f(G ; 1,2,0)=\log _{2} f(G ; 1,0,2)$.

(ii) $p(G)=\log _{2} f(G ; 2,0,1)$.

(iii) $\omega(G)=\log _{2} f(G ; 2,1,0)$.

The edge-connectivity of a graph $G$ is the minimum number $n$ such that there are $n$ edges of $G$ whose removal disconnects $G$ and is denoted by $\lambda(G)$. For example, a connected graph $G$ has a cut edge if and only if $\lambda(G)=1$. Since $b_{12}$ is the number of cut edges of $G$ by Theorem 1.4, $\lambda(G)=1$ if and only if $b_{12}>0$ and $b_{02}=0$ (equivalently $b_{01}=1$ ). In general, the edge-connectivity $\lambda(G)$ can be given by the following, but it is not so easy to decide: the (vertex-) connectivity $\kappa(G)$ by only $f(G)$ :

\section{THEOREM 2.3 (THE EDGE-CONNECTIVITY).}

$$
\lambda(G)=\min \left\{k: b_{k 2}>0\right\} .
$$

There are several classes of graphs which can be characterized by only $p(G)$, $q(G)$ and $\omega(G)$. We can read from $f(G)$ how many such graphs a given graph $G$ contains as its subgraphs. For example, a graph $H$ is tree if and only if $H$ is connected $(\omega(H)=1)$ and has precisely $p(H)-1$ edges $(q(H)=p(H)-1)$. 
THEOREM 2.4 (SPANNING TREES AND FORESTS). (i) $b_{q-p+1} 1=$ the number of spanning trees of $G$.

(ii) $b_{q-p+r} r=$ the number of spanning forests of $G$ with precisely $r$ components.

ProOF. Let $Y$ be any subset of $E(G)$. The spanning subgraph $G-Y$ of $G$ is a forest with precisely $r$ components if and only if $G-Y$ has exactly $p(G)-r$ edges, that is, $|Y|=q(G)-p(G)+r$. By Theorem 1.4, $b_{q-p+r} r$ is the number of such $Y$ 's.

TheOrem 2.5 (COMPlete SUBGRAPHS). Let $G$ be a simple graph. Then, $b_{n(n-1) / 2}{ }_{p-n+1}=$ the number of complete subgraphs of $G$ with precisely $n$ vertices.

ProOF. It is not so difficult to show that a graph $H$ is isomorphic to the disjoint union $K_{n} \cup \overline{K_{p-n}}$, that is, $K_{n}$ with extra $p-n$ isolated vertices if and only if $p(H)=p, q(H)=n(n-1) / 2$ and $\omega(H)=p-n+1$. For $K_{n} \cup \overline{K_{p-n}}$ can be characterized as the unique simple graph which maximizes the number of edges among graphs with the same number of components. Clearly, there is a one-to-one correspondence between complete subgraphs of $G$ with $n$ vertices and spanning subgraphs of $G$ isomorphic to $K_{n} \cup \overline{K_{p-n}}$.

Self-loops are edges which have no effect on the edge-connectivity of a graph. Since a self-loop can be regarded as a spanning subgraph with $p$ components and one edge, the number of self-loops is equal to the coefficient $b_{q-1} p$ of $f(G)$. In fact, their number has an effect on the form of $f(G)$ rather than its coefficients. Notice that $f(G)$ depends on only the number of self-loops but not on how they attach, as follows:

THEOREM 2.6 (SELF-LOOPS). A graph $G$ has $m$ self-loops if and only if $(x+y)^{m}$ divides $f(G)$. Especially, $f(G) /(x+y)^{m}$ is the polynomial of $G$ with $m$ self-loops deleted.

PROOF. If an edge $e$ is a loop, then

$$
f(G)=x f(G / e)+y f(G-e)=(x+y) f(G-e)
$$

since $G / e=G-e$ by our definition. Conversely suppose that $x+y$ divides $f(G)$, then $b_{q-1} p \geq 1$ since $b_{q p}=1$. This means that there is an edge $e \in E(G)$ such that $G-(E(G)-\{e\})$ has precisely $p$ components and hence each vertex has to be isolated in $G-(E(G)-\{e\})$. Thus $e$ is a loop incident to one vertex. Repeat this argument $m$ times to get the theorem.

THEOREM 2.7 (SIMPLENESS). A graph $G$ is simple if and only if $b_{q-1} p+$ $b_{q-2} p-1>0$.

PROOF. The number of self-loops coincides precisely with $b_{q-1} p$ but the number of pairs of multiple edges may not be equal to $b_{q-2} p-1$. For a subgraph with one component and two edges is either a cycle of length 2 or $K_{2}$ with a self-loop at one vertex. In any way, $b_{q-1} p+b_{q-2} p-1>0$ if and only if $G$ has a self-loop or multiple edges.

A vertex coloring $\xi$ of a graph $G$ with $n$ colors is a map $\xi: V(G) \rightarrow\{1,2, \ldots, n\}$ such that $\xi(u) \neq \xi(v)$ for any two adjacent vertices $u$ and $\nu$. It is well-known that the number of vertex colorings of $G$ with $n$ colors can be expressed uniformly as a polynomial in $n[\mathbf{1}]$. We call such a polynomial with variable $t$ the chromatic 
polynomial of $G$ and denote it by $P(G ; t)$. If one assigns a natural number $n$ to $t$, then the value of $P(G ; n)$ gives the total number of vertex colorings of $G$ with at most $n$ colors. In particular, if $G$ is not $n$-colorable, then $P(G ; n)=0$.

Theorem 2.8 (The Chromatic Polynomial).

$$
f(G ; t,-1,1)=P(G ; t) .
$$

Proof. Let $G$ be a graph with at least one edge $e$. If the edge $e$ is not a loop, then we have the formula

$$
P(G ; t)=-P(G / e ; t)+P(G-e ; t) .
$$

For $P(G / e ; t)$ counts the number of vertex colorings of $G-e$ which assign the same colors to the both ends of $e$. If $e$ is a loop, then there is no vertex coloring of $G$ and hence $P(G ; t)=0$. Since $G / e=G-e$ by our definition, the above formula justifies it.

The formula (ii) in the definition of $f(G)$ has the same style as the above if we assign -1 and 1 to $x$ and $y$, respectively. As the initialization, we have

$$
P\left(\overline{K_{n}} ; t\right)=t^{n}=f\left(\overline{K_{n}} ; t,-1,1\right)
$$

since every vertex coloring of $\overline{K_{n}}$ assigns a color to each vertex independently. Therefore $f(G ; t,-1,1)=P(G ; t)$.

Let $D$ be a directed graph with underlying undirected graph $G$ and let $\eta: E(D)$ $\rightarrow A$ be a function from $E(D)$ to an additive group $A$ with identity 0 . We define $\mu(\eta ; \nu)$ for each vertex $\nu \in V(D)=V(G)$ by

$$
\mu(\eta ; \nu)=\sum_{e: \text { incoming }} \eta(e)-\sum_{d: \text { outgoing }} \eta(d) .
$$

If $\eta$ satisfies Kirchhoff's Law, that is, if $\mu(\eta ; \nu)=0$ for all $\nu \in V(G)$, then $\eta$ is called a flow over $A$ or an $A$-flow of $G$. Especially, if $\eta(E(D)) \subset A-\{0\}$, then $\eta$ is said to be nowhere zero. Two $A$-flows $\eta$ and $\eta^{\prime}$ of $G$, associated with two directed graphs $D$ and $D^{\prime}$, are regarded to be identical if for every edge $e$ of $G, \eta(e)=\eta^{\prime}(e)$ or $\eta(e)=-\eta^{\prime}(e)$ according to whether or not $e$ has the same direction in $D$ and $D^{\prime}$.

The flow polynomial $F(G ; t)$ of $G$ is a polynomial in $t$ which gives the total number of nowhere zero flows of $G$ over an additive group $A$ of finite order $|A|=t$. (We can take $\mathbf{Z}_{t}=\{0,1, \ldots, t-1\}$ as $A$.)

\section{THEOREM 2.9 (THE FLOW POLYNOMIAL).}

$$
f(G ; t, t,-1)=t^{p} \cdot F(G ; t)
$$

PROOF. Let $e=u v$ be a directed edge of a directed graph $D$ from $u$ to $\nu$. Suppose that $e$ is not a loop. Given a nowhere zero $A$-flow $\eta^{\prime}: E(D / e) \rightarrow A$ of $D / e$, we define a function $\eta^{\prime \prime}: E(D-e) \rightarrow A$ by $\eta^{\prime \prime}(d)=\eta^{\prime}(d)$. Then $\mu\left(\eta^{\prime \prime} ; s\right)=0$ for every vertex $s \in V(D)$ except $u$ and $\nu$. This function $\eta^{\prime \prime}$ is a nowhere zero $A$-flow of $D-e$ if and only if $\mu\left(\eta^{\prime \prime} ; u\right)=0$ (and necessarily $\mu\left(\eta^{\prime \prime} ; v\right)=0$ ). If $\mu\left(\eta^{\prime \prime} ; u\right)>0$, then we can define a nowhere zero $A$-flow $\eta: E(D) \rightarrow A$ by

$$
\eta(d)= \begin{cases}\eta^{\prime \prime}(d) & (d \neq e), \\ \mu\left(\eta^{\prime \prime} ; u\right) & (d=e) .\end{cases}
$$


Thus, we have the following formula on the number of flows:

$$
F(G ; t)=F(G / e ; t)-F(G-e ; t) .
$$

If $e$ is a loop, then we can construct a nowhere zero $A$-flow of $G$ from a nowhere zero $A$-flow of $G-e$ by assigning any nonzero value in $A$ to $e$. This implies that

$$
F(G ; t)=(t-1) F(G-e ; t) .
$$

Now we shall show the theorem by induction on the number of edges. When a graph $G$ has no edge, then $G$ admits a unique flow, which is empty but is not zero. Thus,

$$
f\left(\overline{K_{n}} ; t, t,-1\right)=t^{n}=t^{n} \cdot F\left(\overline{K_{n}} ; t\right) .
$$

Suppose that the theorem holds for every graph with fewer edges than $G$. If an edge $e$ of $G$ is not a loop, then

$$
\begin{aligned}
f(G ; t, t,-1) & =t f(G / e ; t, t,-1)-f(G-e ; t, t,-1) \\
& =t \cdot t^{p-1} \cdot F(G / e ; t)-t^{p} \cdot F(G-e ; t) \\
& =t^{p}(F(G / e ; t)-F(G-e ; t)) \\
& =t^{p} \cdot F(G ; t)
\end{aligned}
$$

by the definition of $f(G)$ and the above formula for $F(G ; t)$. If $e$ is a loop, then

$$
f(G ; t, t,-1)=(t-1) f(G-e)=t^{p}(t-1) F(G-e ; t)=t^{p} \cdot F(G ; t) .
$$

Corollary 2.10 (EUlerian). A graph $G$ is eulerian if and only if $b_{01}=1$ and $f(G ; 2,2,-1) \neq 0$ (necessarily $\left.=2^{p}\right)$.

ProOF. The value of $f(G ; 2,2,-1) / 2^{p}$ is equal to the number of nowhere zero $\mathbf{Z}_{2}$-flows of $G$ by Theorem 2.9. Thus, when $f(G ; 2,2,-1) \neq 0$, there is a unique flow $\eta$ which assigns $1 \in \mathbf{Z}_{2}$ to each edge of $G$ and each vertex of $G$ must have even degree by Kirchhoff's Law for $\eta$. Since an eulerian graph is connected, we need the condition $b_{01}=1$.

Tutte [3] has defined a $V$-function $g(G)$ as an assignment of some ring elements to graphs with the following three axioms.

(i) $g(\varnothing)=1$.

(ii) If $G$ is a union of two disjoint graphs $K$ and $H$, then

$$
g(G)=g(K) g(H) .
$$

(iii) If $e \in E(G)$ is any edge of $G$ which is not a loop, then

$$
g(G)=g(G / e)+g(G-e) .
$$

This definition is very similar to ours. In fact, $f(G)$ satisfies the above (i) and (ii). If one set $x=y=1$, then (ii) in the definition of $f(G)$ is reduced to (iii) above, but our definition does not ask whether or not an edge $e$ is a loop. To give a $V$-function $g(G)$, we have to set the value of $g(G)$ for each one-vertex graph $G$ with loops.

As one of more general $V$-functions, Tutte defined the dichromatic polynomial $Q(G ; t, z)$ by

$$
Q(G ; t, z)=\sum_{Y \subset E(G)} t^{\omega(G-Y)} z^{\beta(G-Y)}
$$


Also the dichromate or the Tutte polynomial $T(G ; x, y)$ is another $V$-function and is related to $Q(G ; t, z)$ by

$$
T(G ; x, y)=(x-1)^{-\omega(G)} Q(G ; x-1, y-1) .
$$

The chromatic polynomial and the flow polynomial are not $V$-functions but are derived from $Q(G ; t, z)$ as well as Theorems 2.8 and 2.9 .

Comparing Theorem 1.3 with the above expansion of $Q(G ; t, z)$, we can show the following relationship among $Q(G ; t, z), T(G ; x, y)$ and our $f(G)$.

THEOREM 2.11. (i) (The dichromatic polynomial)

$$
f(G ; t z, 1, z)=z^{p} Q(G ; t, z) .
$$

(ii) (The Tutte polynomial)

$$
f(G ;(x-1)(y-1), 1, y-1)=(y-1)^{p}(x-1)^{\omega(G)} T(G ; x, y) .
$$

Here, we shall show several examples. By Theorem 1.4, we can know easily $f(G)$ for graphs with simple cutset structure, not carrying out the recursive process. The graphs below can be determined only by $p(G), q(G)$ and $\omega(G)$, so the polynomial $f(G)$ characterizes them.

THEOREM 2.12. (i) A graph $G$ is a tree with $q$ edges if and only if

$$
f(G)=t(x+t y)^{q} .
$$

(ii) A graph $G$ is a cycle of length $q$ if and only if

$$
f(G)=(x+t y)^{q}+(t-1) x^{q} .
$$

(iii) A graph $G$ consists of a single vertex with $q$ self-loops if and only if

$$
f(G)=t(x+y)^{q} .
$$

(iv) A graph $G$ consists of two vertices joining by $q$ multiple edges if and only if

$$
f(G)=t(x+y)^{q}+t(t-1) y^{q} .
$$

3. Dual polynomials. In this section, we shall define another polynomial $f^{*}(G)$ and discuss the relationship between $f(G)$ and $f^{*}(G)$. Also $f^{*}(G)=$ $f^{*}(G ; t, x, y)$ is defined as a polynomial with three variables $t, x, y$ by the following formulas.

(i) $f^{*}\left(\overline{K_{n}}\right)=t^{n}$.

(ii) If an edge $e \in E(G)$ is a loop, then $f^{*}(G)=(x+t y) f^{*}(G-e)$.

(iii) If an edge $e \in E(G)$ is a cut edge, then $f^{*}(G)=(x+y) f^{*}(G / e)$.

(iv) If an edge $e \in E(G)$ is neither a loop nor a cut edge, then

$$
f(G)=x f(G-e)+y f(G / e) .
$$

We call $f^{*}(G)$ the dual polynomial of $G$. Its well-definedness can be proved inductively but such a proof will branch into as many cases as its definition. Then we shall show it directly after some arguments, understanding what is $f^{*}(G)$.

A dual $G^{*}$ of a connected planar graph $G$ embedded on a sphere $S^{2}$ is the graph obtained in the following way; choose a point $r_{i}$ in the interior of each region $R_{i}$ of $G$ as a vertex of $G^{*}$, and join $r_{i}$ and $r_{j}$ with an edge $e^{*}$ when and only when regions $R_{i}$ and $R_{j}$ meet along an edge $e$ of $G$. Thus any dual $G^{*}$ depends on an 
embedding of $G$ and is naturally embedded on $S^{2}$ so that each edge $e^{*}$ intersects the corresponding edge $e$ transversely in a point. We consider a dual of a disconnected planar graph as the disjoint union of duals of its components embedded individually on distinct spheres. Roughly speaking, $(K \cup H)^{*}=K^{*} \cup H^{*}$ for two disjoint graphs $K$ and $H$. (See $[\mathbf{4}]$ for a combinatorial definition of duals.)

The dual polynomial $f^{*}(G)$ is given its name after the following duality. The proof below will tell us why $f^{*}(G)$ is defined as above.

THEOREM 3.1. Let $G$ be a planar graph and $G^{*}$ any dual graph of $G$. Then $f^{*}(G)=f\left(G^{*}\right)$.

PROOF. We shall show the theorem by induction on the number of edges of a planar graph $G$. When $G$ has no edge, then $G \cong \overline{K_{n}}$ and $G^{*} \cong\left(\overline{K_{n}}\right)^{*}=\overline{K_{n}}$ by our definition. Thus, $f(G)=f^{*}(G)=t^{p(G)}$.

Now assume that $G$ and $G^{*}$ are embedded together on spheres component-wise. If an edge $e$ of $G$ is not a cut edge of $G$, then the two sides of $e$ meet two distinct regions $R_{i}$ and $R_{j}$ of $G$, so the deletion of $e$ unifies $R_{i}$ and $R_{j}$. Thus, the dual of $G-e$ coincides with $G^{*} / e^{*}$ where the two ends $r_{i}$ and $r_{j}$ of $e^{*}$ are identified. On the other hand, if $e$ is not a loop, then the contraction of $e$ cuts the contact of $R_{i}$ and $R_{j}$, now possibly identical, along $e$ and hence $(G / e)^{*}=G^{*}-e^{*}$. Therefore, if $e$ is neither a loop nor a cut edge, then

$$
\begin{aligned}
f\left(G^{*}\right) & =x f\left(G^{*} / e^{*}\right)+y f\left(G^{*}-e^{*}\right) \\
& =x f^{*}(G-e)+y f^{*}\left(G^{*} / e\right)=f^{*}(G)
\end{aligned}
$$

by the induction hypothesis and the definitions of $f(G)$ and $f^{*}(G)$.

If an edge $e$ of $G$ is a loop, then the corresponding edges $e^{*}$ is a cut edge of $G^{*}$. Let $K^{*}$ and $H^{*}$ be two disjoint subgraphs of $G^{*}-e^{*}$ such that $G^{*}-e^{*}=K^{*} \cup H^{*}$ and $e^{*}$ joints $K^{*}$ to $H^{*}$. Then we have

$$
\begin{aligned}
f\left(G^{*}\right) & =x f\left(G^{*} / e^{*}\right)+y f\left(G^{*}-e^{*}\right)=x f\left(K^{*} \cdot H^{*}\right)+y f\left(K^{*} \cup H^{*}\right) \\
& =x f\left(K^{*}\right) f\left(H^{*}\right) / t+y f\left(K^{*}\right) f\left(H^{*}\right) \\
& =(x+t y) f\left(K^{*}\right) f\left(H^{*}\right) / t=(x+t y) f\left(K^{*} \cdot H^{*}\right) .
\end{aligned}
$$

Since $K^{*} \cdot H^{*}=G^{*} / e^{*}$, we conclude that

$$
f\left(G^{*}\right)=(x+t y) f\left(G^{*} / e^{*}\right)=(x+t y) f^{*}(G-e)=f^{*}(G) .
$$

If $e$ is a cut edge of $G$, then $e^{*}$ is a loop of $G^{*}$, and we have

$$
f\left(G^{*}\right)=(x+y) f\left(G^{*}-e^{*}\right)=(x+y) f^{*}(G / e)=f^{*}(G)
$$

since $f\left(G^{*} / e^{*}\right)=f\left(G^{*}-e^{*}\right)$.

Now we shall consider a resolution of a graph $G$ for calculating $f^{*}(G)$. Let $W$ be a tree-like set of words in $x$ and $y$. A family

$$
R^{*}(G ; W)=\{G(W): w \in W\}
$$

with $G(\varnothing)=G$ is called a dual resolution if one of the following three (i), (ii), (iii) holds for each word $w \in W-\Omega(W)$ :

(i) For some loop $e(w) \in E(G(w))$,

$$
G(w x)=G(w y)=G(w)-e(w) .
$$


(ii) For some cut edge $e(w) \in E(G(w))$,

$$
G(w x)=G(w y)=G(w) / e(w) .
$$

(iii) For some edge $e(w) \in E(G(w))$ which is neither a loop nor a cut edge,

$$
G(w x)=G(w)-e(w), \quad G(w y)=G(w) / e(w) .
$$

We define the terms "total", "partial", "standard" and "depth" for dual resolutions as well as those for resolutions previously defined.

When we calculate $f^{*}(G)$ practically, we had better not make two branches under each stage with $e(w)$ a loop or a cut edge. We make only one branch and assign a weight $(x+t y)$ or $(x+y)$ to it when $e(w)$ is a loop or a cut edge, respectively. Figure 2 shows a standard total dual resolution for $C_{3}$ in the practical sense and suggests the relationship between it and a resolution for the dual of $C_{3}$.

Considering a dual resolution, we shall show the following expansion formula for $f^{*}(G)$, which implies the well-definedness of $f^{*}(G)$.

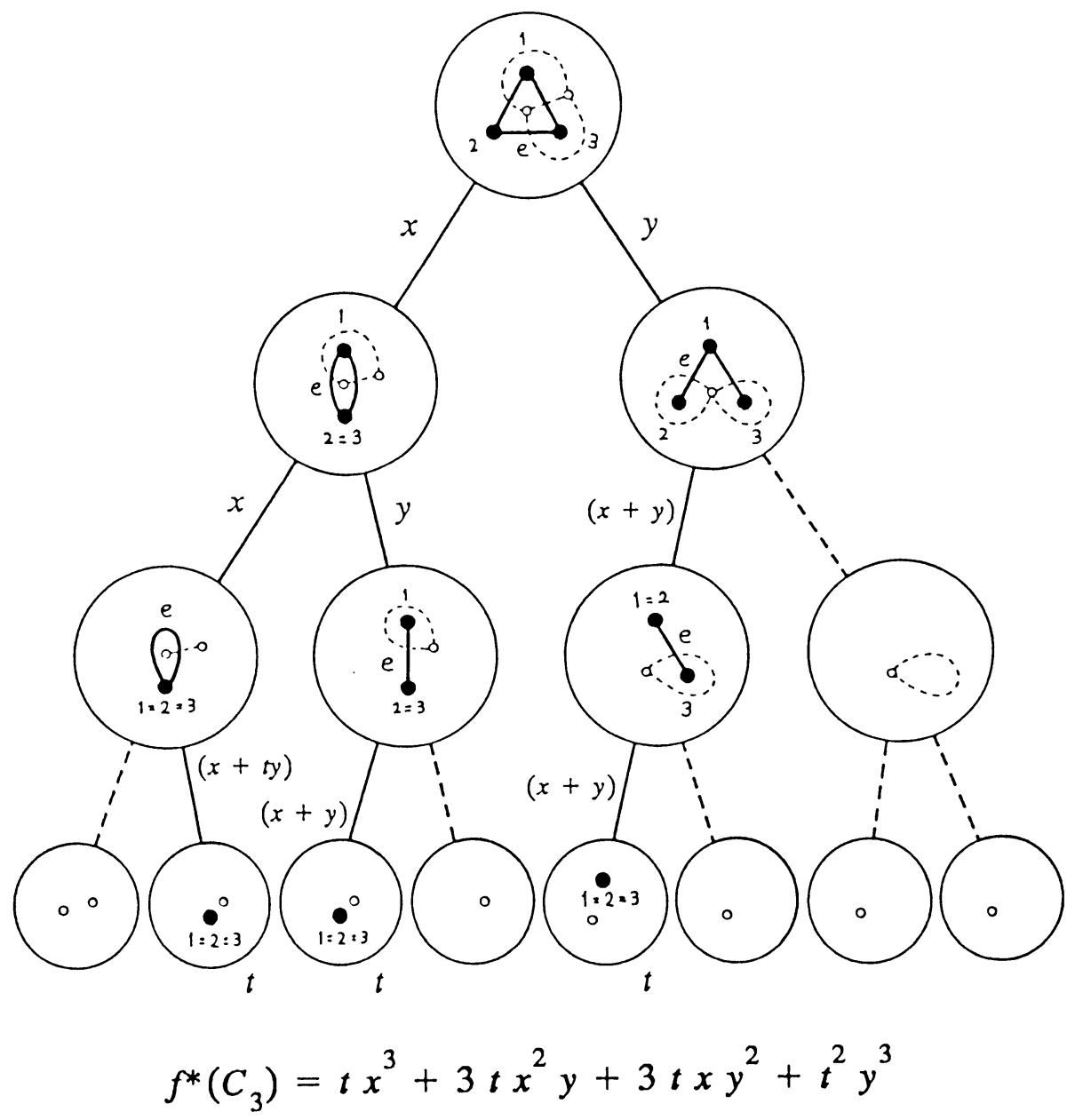

Figure 2. A dual resolution of $C_{3}$. 
THEOREM 3.2. $f^{*}(G)=t^{\omega}\left(\sum_{X \subset E(G)} t^{\beta(G-X)} x^{|X|} y^{q-|X|}\right)$.

Proof. Let $R^{*}(G ; W)=\{G(w): w \in W\}$ be a standard total dual resolution of a graph $G$ with edges $e_{1}, \ldots, e_{q}$. Let $w=l_{1} \cdots l_{q} \in \Omega(W)\left(l_{i} \in\{x, y\}\right)$ be any end word and $w_{k}=l_{1} \cdots l_{k}(1 \leq k \leq q)$ its subword of length $k$. We define four disjoint subsets of $E(G)$ as follows:

$$
\begin{aligned}
& X_{0}(w)=\left\{e_{k} \in E(G): l_{k}=x, e_{k} \text { is not a cut edge in } G\left(w_{k}\right)\right\}, \\
& X_{1}(w)=\left\{e_{k} \in E(G): l_{k}=x, e_{k} \text { is a cut edge in } G\left(w_{k}\right)\right\}, \\
& Y_{0}(w)=\left\{e_{k} \in E(G): l_{k}=y, e_{k} \text { is not a loop in } G\left(w_{k}\right)\right\}, \\
& Y_{1}(w)=\left\{e_{k} \in E(G): l_{k}=y, e_{k} \text { is a loop in } G\left(w_{k}\right)\right\} .
\end{aligned}
$$

Then $G(w)=\left(G-\left(X_{0}(w) \cup Y_{1}(w)\right)\right) /\left(X_{1}(w) \cup Y_{0}(w)\right)$. It is clear that $f^{*}(G)$ expands as

$$
\begin{aligned}
f^{*}(G) & =\sum_{w \in \Omega(W)} t^{p(G(w))} x^{|X(w)|} y^{\left|Y_{0}(w)\right|}(t y)^{\left|Y_{1}(w)\right|} \\
& =\sum_{w \in \Omega(W)} t^{p(G(w))+\left|Y_{1}(w)\right|} x^{|X(w)|} y^{|Y(w)|}
\end{aligned}
$$

where $X(w)=X_{0}(w) \cup X_{1}(w)$ and $Y(w)=Y_{0}(w) \cup Y_{1}(w)=E(G)-X(w)$. Since $X_{0}(w) \cup Y_{1}(w)$ contains no cut edge, $G$ and $G(w)$ have the same number $\omega$ of components. On the other hand, $Y_{0}(w)$ induces a spanning forest of $G-X(w)$ with isolated points neglected, so $\left|Y_{1}(w)\right|=\beta(G-X(w))$. Since $X(w)$ ranges over all subsets of $E(G)$, the above expansion implies the theorem.

Comparing Theorem 3.2 with Theorem 1.3, we get the following relationship between $f(G)$ and $f^{*}(G)$.

THEOREM 3.3. $t^{\omega-p} f(G ; t, t y, x)=f^{*}(G ; t, x, y)$.

Therefore,

COROllary 3.4. For two graphs $G$ and $G^{\prime}, f(G)=f\left(G^{\prime}\right)$ if and only if $f^{*}(G)=f^{*}\left(G^{\prime}\right)$.

The product formulas below hold for $f^{*}(G)$ as well as Theorem 1.5. They follow immediately from Theorems 1.5 and 3.3 and also can be proved inductively.

THEOREM 3.5. (i) If $K$ and $H$ are disjoint, then

$$
f^{*}(K \cup H)=f^{*}(K) f^{*}(H) .
$$

(ii) If $K$ and $H$ intersect in exactly one vertex, then

$$
f^{*}(K \cdot H)=f^{*}(K) f^{*}(H) / t
$$

4. Splitting formulas. In this section, we shall discuss how the polynomial $f(G)$ can be recognized when a graph $G$ splits into two subgraphs. Let $K$ and $H$ be two edge-disjoint graphs which meet in $n$ vertices and set $G=K \cup H$ hereafter.

Let $U=\left\{u_{1}, u_{2}, \ldots, u_{n}\right\}$ be the set of common vertices of $K$ and $H$. We denote a partition of $U$ as a sequence divided by semicolons. For example, $\gamma=$ $\left(u_{2} ; u_{1}, u_{3} ; u_{4}, u_{5}, u_{6}\right)$ represents the partition of $U$ into three subsets $U_{1}=\left\{u_{2}\right\}$, $U_{2}=\left\{u_{1}, u_{3}\right\}$ and $U_{3}=\left\{u_{4}, u_{5}, u_{6}\right\}$ and we also write $\gamma=\left(U_{1} ; U_{2} ; U_{3}\right)$. The number of subsets $U_{k}$ in a partition $\gamma$ is called the size of $\gamma$ and is denoted by $|\gamma|$. 
Let $\Gamma(U)$ be the set of all partitions of $U$. We define an order " $\leq$ " over $\Gamma(U)$ so that $\gamma \leq \gamma^{\prime}$ if and only if $\gamma^{\prime}=\left(U_{1}^{\prime}, \ldots, U_{l}^{\prime}\right)$ is a refinement of $\gamma=\left(U_{1}, \ldots, U_{m}\right)$, that is, each $U_{h}^{\prime}$ is contained in some $U_{k}$. Then this order makes $\Gamma(U)$ be a lattice, called the partition lattice. We denote the supremum and the infimum of two partitions $\gamma$ and $\gamma^{\prime}$ in $\Gamma(U)$ by $\gamma \vee \gamma^{\prime}$ and $\gamma \wedge \gamma^{\prime}$, respectively. In particular, two elements $u_{i}$ and $u_{j}$ of $U$ belong to a common part of $\gamma \wedge \gamma^{\prime}$ if and only if there is an alternating sequence of $U_{k}$ and $U_{h}^{\prime}$ such that its origin and terminus contain $u_{i}$ and $u_{j}$, respectively, and successive $U_{k}$ and $U_{h}^{\prime}$ have nonempty intersection.

It is obvious that if $\gamma \leq \gamma^{\prime}$ then $|\gamma| \leq\left|\gamma^{\prime}\right|$. We give each partition a subscript $i \in$ $\{1,2, \ldots,|\Gamma(U)|\}$ so that if $\gamma_{i} \leq \gamma_{j}$ in $\Gamma(U)$ then $i \leq j$. Then $\gamma_{1}=\left(u_{1}, u_{2}, \ldots, u_{n}\right)$ and $\gamma_{|\Gamma(U)|}=\left(u_{1} ; u_{2} ; \ldots ; u_{n}\right)$.

Let $T_{n}$ denote the $|\Gamma(U)| \times|\Gamma(U)|$ matrix with $(i, j)$-entries $t^{\left|\gamma_{i} \wedge \gamma_{j}\right|} \in \mathbf{Q}(t)$. For example, $T_{1}=t$. When $n=2$, there are only two partitions of $U=\left\{u_{1}, u_{2}\right\}$, namely $\left(u_{1}, u_{2}\right)$ and $\left(u_{1} ; u_{2}\right)$. Thus,

$$
T_{2}=\left(\begin{array}{cc}
t & t \\
t & t^{2}
\end{array}\right)
$$

and it has the inverse

$$
T_{2}^{-1}=\frac{1}{t(t-1)}\left(\begin{array}{cc}
t & -1 \\
-1 & 1
\end{array}\right)
$$

When $n=3$, there are precisely five partitions of $U=\left\{u_{1}, u_{2}, u_{3}\right\}: \gamma_{1}=$ $\left(u_{1}, u_{2}, u_{3}\right), \gamma_{2}=\left(u_{1} ; u_{2}, u_{3}\right), \gamma_{3}=\left(u_{2} ; u_{1}, u_{3}\right), \gamma_{4}=\left(u_{3} ; u_{1}, u_{2}\right)$ and $\gamma_{5}=$ $\left(u_{1} ; u_{2} ; u_{3}\right)$.

$$
\begin{aligned}
& T_{3}=\left(\begin{array}{ccccc}
t & t & t & t & t \\
t & t^{2} & t & t & t^{2} \\
t & t & t^{2} & t & t^{2} \\
t & t & t & t^{2} & t^{2} \\
t & t^{2} & t^{2} & t^{2} & t^{3}
\end{array}\right), \\
& T_{3}^{-1}=\frac{1}{t(t-1)(t-2)}\left(\begin{array}{cccccc}
t^{2} & -t & -t & -t & 2 \\
-t & t-1 & 1 & 1 & -1 \\
-t & 1 & t-1 & 1 & -1 \\
-t & 1 & 1 & t-1 & -1 \\
2 & -1 & -1 & -1 & 1
\end{array}\right) .
\end{aligned}
$$

In general, every $T_{n}(n \geq 1)$ has the inverse. Only routine work is needed to get each $T_{n}^{-1}$, but it will be so difficult to show its general form:

LEMMA 4.1. The square matrix $T_{n}=\left(t^{\left|\gamma_{i} \wedge \gamma_{j}\right|}\right)$ is regular.

Proof. The determinant $\operatorname{det}\left(T_{n}\right)$ is a polynomial in $t$ and its degree is attained by only the diagonal product $\prod_{\gamma_{i} \in \Gamma(U)} t^{\left|\gamma_{i} \wedge \gamma_{i}\right|}$. So the other products cannot eliminate this factor in the expansion of $\operatorname{det}\left(T_{n}\right)$. Therefore, $\operatorname{det}\left(T_{n}\right) \neq 0$.

For any partition $\gamma_{i}=\left(U_{1} ; \ldots ; U_{s}\right)$ of $U$, we define $K / \gamma_{i}$ as the graph obtained from $K$ by identifying all vertices in each $U_{k}$ to a single vertex. For example, the $n$ vertices are regarded as one vertex in $K / \gamma_{1}$ and $K / \gamma_{|\Gamma(U)|}=K$. Similarly, we define $H / \gamma_{i}$. 
Let ${ }^{t} \mathbf{f}(K / \Gamma(U))$ and $\mathbf{f}(H / \Gamma(U))$ denote the line and column vectors, respectively, defined by

$$
\begin{gathered}
{ }^{t} \mathbf{f}(K / \Gamma(U))=\left(f\left(K / \gamma_{1}\right), f\left(K / \gamma_{2}\right), \ldots, f\left(K / \gamma_{|\Gamma(U)|}\right)\right), \\
\mathbf{f}(H / \Gamma(U))=\left(\begin{array}{c}
f\left(H / \gamma_{1}\right) \\
f\left(H / \gamma_{2}\right) \\
\vdots \\
f\left(H / \gamma_{|\Gamma(U)|}\right)
\end{array}\right) .
\end{gathered}
$$

Then we have the following formula for $f(G)$.

THEOREM 4.2 (SPLITTING FORMULA). Let $G$ be a graph obtained as a union of two graphs $K$ and $H$ which meet in $U=\left\{u_{1}, \ldots, u_{n}\right\}$. Then

$$
f(G)={ }^{t} \mathbf{f}(K / \Gamma(U)) T_{n}^{-1} \mathbf{f}(H / \Gamma(U)) .
$$

Proof. Consider any total resolution $R(H ; W)=\{H(w): w \in W\}$ of $H$. Then $f(H(w))=t^{p(H(w))}$ for each end word $w \in \Omega(W)$ since $H(w)$ consists of only $p(H(w))$ isolated vertices. Let $\gamma_{i}=\left(U_{1}, \ldots, U_{s}\right)$ be a partition of $U$ of size $\left|\gamma_{i}\right|=s$ and let $A\left(\gamma_{i}\right)$ be the sum up of $[w] f(H(w)) / t^{s}$ for all $H(w)(w \in \Omega(W))$ such that the vertices in each $U_{k}(1 \leq k \leq m)$ are identified to one vertex in $H(w)$. Then we have

$$
f(H)=\sum_{\gamma_{i} \in \Gamma(U)} A\left(\gamma_{i}\right) t^{\left|\gamma_{i}\right|} .
$$

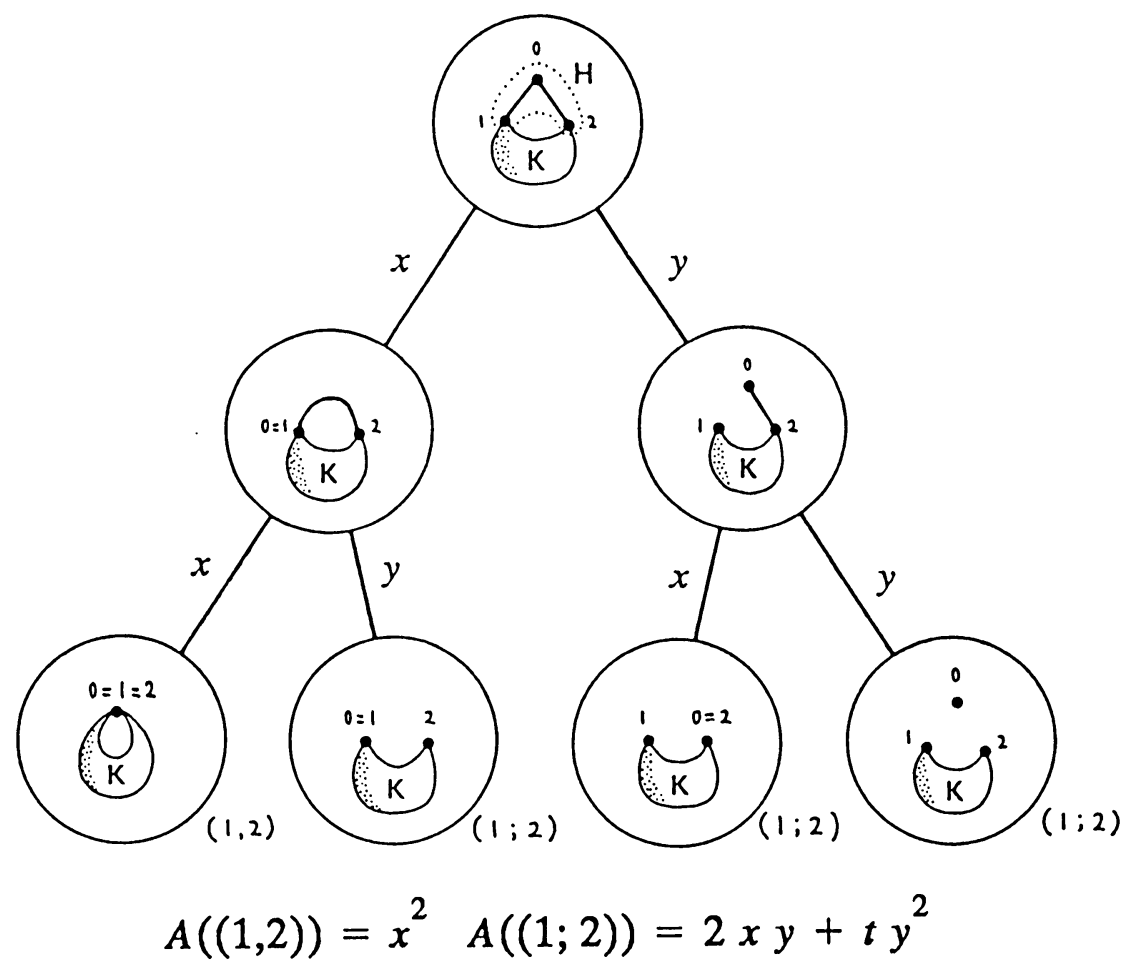

Figure 3. A resolution for $K \cup H$. 
Now consider the partial resolution $R(G ; W)=\{G(w): w \in W\}$ of depth $q(H)$ for $G$, in parallel to $R(H ; W)$ with the same tree-like set $W$. (See Figure 3.) Each graph $G(w)(w \in W)$ looks like $H(w)$ with $K$ added, where the vertices of $K$ in $U$ are suitably identified with one another. In particular, each end graph $G(w)=H(w) \cup K / \gamma_{i}(w \in \Omega(W))$ can be obtained by replacing $\left|\gamma_{i}\right|$ vertices of $H(w)$ with $K / \gamma_{i}$ for some $\gamma_{i} \in \Gamma(U)$. Thus,

$$
f(G)=\sum_{\gamma_{i} \in \Gamma(U)} A\left(\gamma_{i}\right) f\left(K / \gamma_{i}\right) .
$$

If we substitute $H / \gamma_{j}$ for $H$ in $R(H ; W)$ and deform it in the same process of deletion and contraction of edges, we will get a total resolution $R\left(H / \gamma_{j} ; W\right)$ and conclude the following formula, similar to (1), for $f\left(H / \gamma_{i}\right)$ :

$$
f\left(H / \gamma_{j}\right)=\sum_{\gamma_{i} \in \Gamma(U)} A\left(\gamma_{i}\right) t^{\left|\gamma_{i} \wedge \gamma_{j}\right|} .
$$

Since $T_{n}$ has inverse, equation (3) is solvable with respect to $A\left(\gamma_{i}\right)$ 's. Substituting the solution to (2), we can write down $f(G)$ in terms of only $f\left(K / \gamma_{i}\right)$ and $f\left(H / \gamma_{j}\right)$ as the theorem shows.

When $n=1$, the splitting formula is nothing but Theorem 1.5(ii). The following formula is the explicit form of the splitting formula with $n=2$.

COROLLARY 4.3. If $K$ and $H$ meet at precisely two vertices $u_{1}$ and $u_{2}$, then

$$
\begin{aligned}
f(G)=\left\{\left(t f\left(H /\left(u_{1}, u_{2}\right)\right)\right.\right. & -f(H)) f\left(K /\left(u_{1}, u_{2}\right)\right) \\
& \left.-\left(f\left(H /\left(u_{1}, u_{2}\right)\right)-f(H)\right) f(K)\right\} / t(t-1) .
\end{aligned}
$$

We wrote the splitting formula in the Introduction as

$$
f(G)=\sum_{(\gamma, \delta) \in \Gamma(U) \times \Gamma(U)} f(K / \gamma) B_{n}(\gamma, \delta ; t) f(H / \delta) .
$$

Comparing this with Theorem 4.2 , we see that $B_{n}\left(\gamma_{i}, \gamma_{j} ; t\right)$ is the $(i, j)$-entry of $T_{n}^{-1}$. The rational functions $B_{n}(\gamma, \delta ; t)$ 's are called the $n$th pasting coefficients for $f(G)$.

Also the coefficients of $X(K / \gamma) X(H / \delta)$ 's in the same type expansion for any other polynomial $X(G)$ will be called pasting coefficients for $X(G)$. The polynomial $X(G)$ is said to split nicely if the pasting coefficients do not depend on the structure of $K$ and $H$. Then our polynomial $f(G)$ splits nicely in this sense.

We can translate the splitting formula for $f(G)$ into that for $f^{*}(G)$ via Theorem 3.3 as below. The power of $t$ in the last formula however depends on the structures of $K$ and $H$. So $f^{*}(G)$ does not split so nicely as $f(G)$ in general.

$$
\begin{aligned}
f^{*}(G ; t, x, y)= & t^{\omega(G)-p(G)} f(G ; t, t y, x) \\
= & t^{\omega(G)-p(G)} \sum f(K / \gamma ; t, t y, x) B_{n}(\gamma, \delta ; t) f(H / \delta ; t, x, y) \\
= & \sum\left[B_{n}(\gamma, \delta ; t) t^{(\omega(G)-\omega(K / \gamma)-\omega(H / \delta))-(p(G)-p(K / \gamma)-p(H / \delta))}\right] \\
& \times f^{*}(K / \gamma ; t, x, y) f^{*}(H / \delta ; t, x, y) \\
= & \sum\left[B_{n}(\gamma, \delta ; t) t^{(\omega(G)-\omega(K / \gamma)-\omega(H / \delta))-(n-|\gamma|-|\delta|)}\right] \\
& \times f^{*}(K / \gamma ; t, x, y) f^{*}(H / \delta ; t, x, y)
\end{aligned}
$$


So as to get a nice splitting formula for $f^{*}(G)$, we restrict all $G, K$ and $H$ to be connected. In this case, the factor between brackets does not depend on $K$ and $H$ since

$$
\omega(G)-\omega(K / \gamma)-\omega(H / \delta)=1-1-1=-1
$$

Therefore, we get the following formula.

THEOREM 4.4 (SPLITTING FORMULA FOR $f^{*}(G)$ ). Let $G$ be a graph obtained as a union of two graphs $K$ and $H$ which meet in $U=\left\{u_{1}, \ldots, u_{n}\right\}$. If $G, K$ and $H$ are connected, then

$$
f^{*}(G)=\sum_{(\gamma, \delta) \in \Gamma(U) \times \Gamma(U)} f^{*}(K / \gamma)\left[B_{n}(\gamma, \delta ; t) t^{|\gamma|+|\delta|-n-1}\right] f^{*}(H / \delta) .
$$

Corollary 4.5. Suppose that $G, K$ and $H$ are connected. If $K$ and $H$ meet at precisely two vertices $u_{1}$ and $u_{2}$, then

$$
\begin{aligned}
f^{*}(G)=\left\{\left(f^{*}\left(H /\left(u_{1}, u_{2}\right)\right)\right.\right. & \left.-f^{*}(H)\right) f^{*}\left(K /\left(u_{1}, u_{2}\right)\right) \\
& \left.-\left(f^{*}\left(H /\left(u_{1}, u_{2}\right)\right)-t f^{*}(H)\right) f^{*}(K)\right\} / t(t-1) .
\end{aligned}
$$

By Theorems 2.8 to 2.11, splitting formulas for the chromatic, flow, dichromatic and Tutte polynomials can be derived from Theorem 4.2 , as follows. The first three split nicely but the Tutte polynomial does not.

COROLlary 4.7 (SPlitTing FORMUlas FOR OTHER POLYNOMIALS). Suppose that $G$ splits into $K$ and $H$ with $U=V(K) \cap V(H)$ as above. Then we have the following formulas, where each summation is taken over all $(\gamma, \delta) \in \Gamma(U) \times \Gamma(U)$ :

(i) (The chromatic polynomial)

$$
P(G ; t)=\sum P(K / \gamma ; t) B_{n}(\gamma, \delta ; t) P(H / \delta ; t) .
$$

(ii) (The flow polynomial)

$$
F(G ; t)=\sum F(K / \gamma ; t)\left[B_{n}(\gamma, \delta ; t) t^{|\gamma|+|\delta|-n}\right] F(H / \delta ; t) .
$$

(iii) (The dichromatic polynomial)

$$
Q(G ; t, z)=\sum Q(K / \gamma ; t, z)\left[B_{n}(\gamma, \delta ; t z) z^{|\gamma|+|\delta|-n}\right] Q(H / \delta ; t, z) .
$$

(iv) (The Tutte polynomial)

$$
\begin{aligned}
T(G ; x, y)= & \sum\left[B_{n}(\gamma, \delta ;(x-1)(y-1))(y-1)^{|\gamma|+|\delta|-n}\right. \\
& \left.\cdot(x-1)^{\omega(K / \gamma)+\omega(H / \delta)-\omega(G)}\right] \\
& \times T(K / \gamma ; x, y) T(H / \delta ; x, y) .
\end{aligned}
$$

We shall end this section with a negative result which warns us not to try establishing a simple splitting formula. Both formulas in Theorem 1.5 have the form

$$
f(G)=f(K) f(H) / f(K \cap H)
$$

since $f(\varnothing)=1$ and $f\left(K_{1}\right)=t$. One might expect such a formula in other cases, 
but the following theorem tells that it is hardly possible:

THEOREM 4.8. There is no graph $F$ with at least two vertices such that for all graph $G$, if $G$ is a union of two graphs $K$ and $H$ whose intersection is isomorphic to $F$ then $f(G)=f(K) f(H) / f(F)$.

Proof. Suppose that $F$ has two distinct vertices $u$ and $v$. Let $G$ be the graph obtained from $F$ and two extra vertices $s$ and $w$ by adding edges $u s, u w, v s$ and $v w$. Then

$$
\begin{aligned}
f(G)= & x f(G / u s)+y f(G-u s) \\
= & x(x f((G / u s) / u w)+y f((G / u s)-u w)) \\
& +y(x f((G-u s) / u w)+y f((G-u s) / u w)) .
\end{aligned}
$$
Then

We denote $F$ with one or two edges joining $u$ and $v$ by $F+e$ or $F+2 e$, respectively.

Thus, we have

$$
\begin{gathered}
(G / u s) / u w \cong F+2 e, \\
(G / u s)-u w \cong(G-u s) / u w \cong(F+e) \cdot K_{2}, \\
(G-u s)-u w \cong F \cdot K_{2} \cdot K_{2} .
\end{gathered}
$$

$$
f(G)=x^{2} f(F+2 e)+2 x y f(F+e) f\left(K_{2}\right) / t+y^{2} f(F) f\left(K_{2}\right)^{2} / t^{2} .
$$

On the other hand, it is easy to show the formulas

$$
\begin{aligned}
f(F+e) & =x f((F+e) / e)+y f(F), \\
f(F+2 e) & =x f((F+e) / e) f\left(K_{1} \text { with a loop }\right) / t+y f(F+e) \\
& =x(x+y) f((F+e) / e)+y(x f((F+e) / e)+y f(F)) \\
& =(x(x+y)+y x) f((F+e) / e)+y^{2} f(F) .
\end{aligned}
$$

Now $G$ decomposes into two graphs $K=(F \cup\{s\})+\{u s, v s\}$ and $H=(F \cup$ $\{w\})+\{u w, v w\}$ for which $K \cap H=F$.

$$
\begin{aligned}
f(K) & =x f(K / u s)+y f(K-u s) \\
& =x f(F+e)+y f(F) f\left(K_{2}\right) / t \\
& =x f(F+e)+y(x+t y) f(F)(=f(H)) .
\end{aligned}
$$

Now we suppose that $f(G)=f(K) f(H) / f(F)$. Substituting this in $f(G) f(F)=$ $f(K)^{2}$, we get

$$
\begin{gathered}
\left\{x^{2} f(F+2 e)+2 x y(x+t y) f(F+e)+y^{2}(x+t y)^{2} f(F)\right\} f(F) \\
=x^{2} f(F+e)^{2}+2 x y(x+t y) f(F+e) f(F)+y^{2}(x+t y)^{2} f(F)^{2} . \\
\therefore \quad f(F+e)^{2}=f(F+2 e) f(F), \\
\{x f((F+e) / e)+y f(F)\}^{2}=\left\{x(x+2 y) f((F+e) / e)+y^{2} f(F)\right\} f(F) .
\end{gathered}
$$

Finally this implies that $f((F+e) / e)=f(F)$. However, $(F+e) / e$ and $F$ have the same number of edges but the numbers of their vertices are different. Thus, their polynomial cannot be equal by Theorem 2.1. The assumption of $F$ having at least 2 vertices causes this contradiction.

If $K \cap H$ is a complete subgraph of $G$, then the chromatic polynomial $P(G ; t)$ can be expressed as $P(K ; t) P(H ; t) / P(K \cap H ; t)$. Therefore Theorem 4.8 implies that there exist infinitely many pairs $\left(G, G^{\prime}\right)$ such that $P(G ; t)=P\left(G^{\prime} ; t\right)$ but $f(G)=f\left(G^{\prime}\right)$. 
5. Graphs with the same polynomials. Here we shall discuss sufficient conditions for two graphs to have the same polynomial. We have already had such a condition for planar graphs. Theorem 3.1 implies that graphs which are dual to a common planar graph have the same polynomial $f(G)$. For example, three graphs in Figure 4 are distinct duals of a two-vertex graph $G$ with three multiple edges and two loops and their polynomials are equal to $f^{*}(G)$. Moving the block in the dashed circle causes the difference among them. Figure 5 shows another situation where turning around two vertices yields two nonisomorphic duals of a planar graph, a pentagon with two pairs of multiple edges and two chords. We shall generalize these phenomena and define the 2-isomorphism of graphs.

Let $G=K \cdot H$ be a one-point join of two graphs $K$ and $H$ with intersection $\{v\}$. Prepare disjoint copies of $K$ and $H$ and join them at a vertex different from $v$. Then we get another one-point join $G^{\prime}$ of $K$ and $H$. This transformation of $G$ into $G^{\prime}$ is called arrangement of blocks. (See Figure 4.)

Now assume that $G$ can be obtained from disjoint $K$ and $H$ by identifying two distinct vertices $u_{1}$ and $u_{2} \in V(K)$ with $v_{1}$ and $v_{2} \in V(H)$, respectively. We call such $G$ a two-point join of $K$ and $H$. Turning around two vertices $\left\{u_{1}, u_{2}\right\}$ $\left(=\left\{v_{1}, v_{2}\right\}\right)$ is to modify the identification so that $u_{1}=v_{2}$ and $u_{2}=v_{1}$. (See Figure 5.)

Two graphs $G$ and $G^{\prime}$ are said to be 2-isomorphic if $G$ can be transformed into $G^{\prime}$ by a finite sequence of arrangement of blocks and turning around two vertices. Whitney [5] showed that two graphs $G$ and $G^{\prime}$ are 2-isomorphic if and only if there is a bijection $\theta: E(G) \rightarrow E\left(G^{\prime}\right)$ which induces a one-to-one correspondence between cycles of $G$ and of $G^{\prime}$. By the matroidal duality, such a map $\theta$ also induces a one-toone correspondence between (minimal) cutsets of $G$ and of $G^{\prime}$. Roughly speaking,
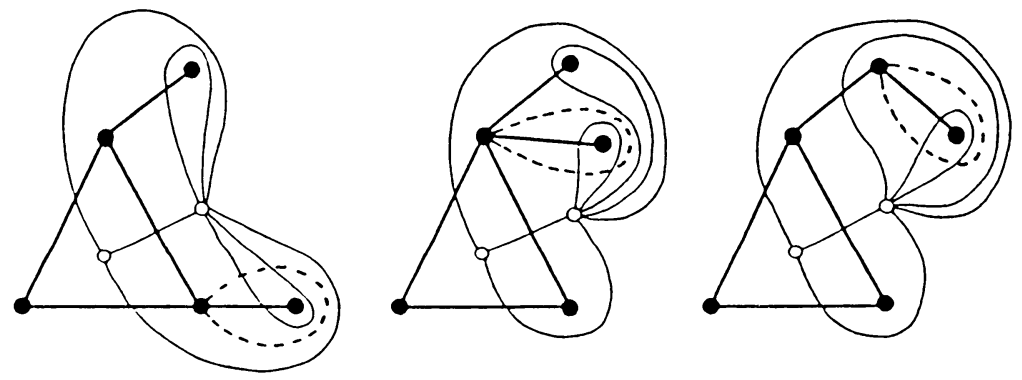

FIGURE 4. Arrangement of blocks.
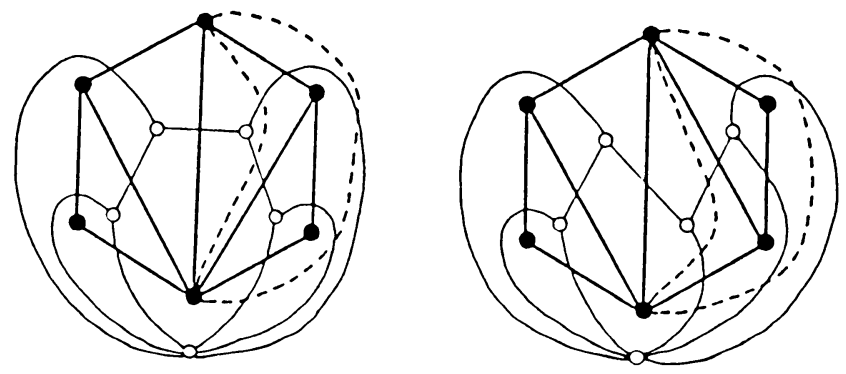

FIGURE 5. Turning around two vertices. 
$f(G)$ can be determined by the number of cutsets. So it will be a natural fact that 2-isomorphic graphs have the same $f(G)$.

THEOREM 5.1. If two graphs $G$ and $G^{\prime}$ are 2-isomorphic, then $f(G)=f\left(G^{\prime}\right)$ and $f^{*}(G)=f^{*}\left(G^{\prime}\right)$.

To prove this, we shall discuss more generally the case when a graph $G=K \cup H$ splits into two edge-disjoint graphs $K$ and $H$ with $n$ vertices $U$ in common, as in the previous section. Then we shall produce another graph from $K$ and $H$ by modifying the joint of $K$ and $H$. Let $\sigma: U \rightarrow U$ be a bijection of $U$ and consider $K$ and $H$ separately. Identify each vertex $u_{i} \in U$ of $K$ with the vertex $\sigma\left(u_{i}\right) \in U$ of $H$. We denoted the resulting graph by $K \cup_{\sigma} H$. Especially, if $\sigma$ is the identity map of $U$, then $K \cup_{\sigma} H$ is nothing but $G$, and if $\sigma$ extends to an automorphism of $H$, then $K \cup_{\sigma} H$ is isomorphic to $G$.

Set $\sigma\left(\gamma_{i}\right)=\left(\sigma\left(U_{1}\right) ; \ldots ; \sigma\left(U_{s}\right)\right)$ for each partition $\gamma_{i}=\left(U_{1} ; \ldots ; U_{s}\right)$ of $U$ and

$$
\mathbf{f}(H / \sigma(\Gamma(U)))=\left(\begin{array}{c}
f\left(H / \sigma\left(\gamma_{1}\right)\right) \\
f\left(H / \sigma\left(\gamma_{2}\right)\right) \\
\vdots \\
f\left(H / \sigma\left(\gamma_{|\Gamma(U)|}\right)\right)
\end{array}\right)
$$

As an immediate consequence of Theorem 4.2, we have the next criterion for two graphs to have the same polynomial:

LEMMA 5.2. If $f\left(H / \gamma_{i}\right)=f\left(H / \sigma\left(\gamma_{i}\right)\right)$ for every partition $\gamma_{i} \in \Gamma(U)$, then $f(G)=f\left(K \cup_{\sigma} H\right)$.

Proof. Sewing $K$ and $H$ by $\sigma: U \rightarrow U$, we identify each partition $\gamma_{i}$ of $U$ in $K$ with the partition $\sigma\left(\gamma_{i}\right)$ of $U$ in $H$. So the splitting formula, Theorem 4.2, for $K \cup_{\sigma} H$ is written as

$$
f\left(K \cup_{\sigma} H\right)={ }^{t} \mathbf{f}(K / \Gamma(U)) T_{n}^{-1} \mathbf{f}(H / \sigma(\Gamma(U))) .
$$

Since $\mathbf{f}(H / \Gamma(U))=\mathbf{f}(H / \sigma(\Gamma(U)))$, we have

$$
\begin{aligned}
f(G)-f\left(K \cup_{\sigma} H\right) & ={ }^{t} \mathbf{f}(K / \Gamma(U)) T_{n}^{-1}(\mathbf{f}(H / \Gamma(U))-\mathbf{f}(H / \sigma(\Gamma(U)))) \\
& =0 .
\end{aligned}
$$

That is, $f(G)=f\left(K \cup_{\sigma} H\right)$.

ProOF OF THEOREM 5.1. Let $G$ be a two-point join of $K$ and $H$ at two vertices $U=\left\{u_{1}, u_{2}\right\}$ and let $\sigma: U \rightarrow U$ be the bijection which interchanges $u_{1}$ and $u_{2}$. Then $K \cup_{\sigma} H$ is the result of turning around $U$. Since $\sigma\left(\gamma_{i}\right)=\gamma_{i}(i=1,2)$, $H / \sigma\left(\gamma_{i}\right)$ is isomorphic to $H / \gamma_{i}$ and the assumption of Lemma 5.2 holds necessarily. Thus, $f(G)=f\left(K \cup_{\sigma} H\right)$.

When $G$ is a one-point join of $K$ and $H$ at $u_{1}$, we regard $G$ as a two-point join of $K$ and $H \cup\left\{u_{2}\right\}$ for a vertex $u_{2}$ in $K$ different from $u_{1}$. Then the one-point join of $K$ and $H$ at $u_{2}$ is the result of turning of $H \cup\left\{u_{2}\right\}$ around $U$. Thus, arrangement of blocks preserves the polynomial $f(G)$. (Also Theorem 1.5(ii) directly implies it.)

Now we shall define an equivalence relation over graphs, weaker than the 2isomorphism. Let $G=K \cup H$ be a graph obtained as a union of two edge-disjoint 

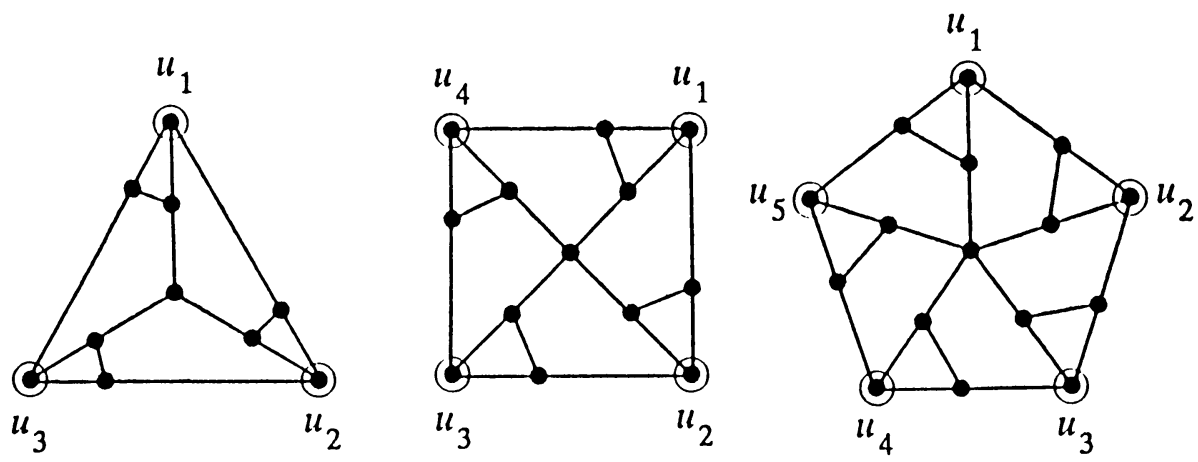

FiguRE 6. Rotors of order $3,4,5$.

graphs $K$ and $H$ with intersection $U=V(K) \cap V(H)$. Let $\sigma: U \rightarrow U$ be a bijection and suppose that

$$
H / \gamma_{i} \cong H / \sigma\left(\gamma_{i}\right) \text { for any partition } \gamma_{i} \text { of } U \text {. }
$$

The graph $G^{\prime}=K \cup_{\sigma} H$, as defined previously, is called the result of an admissible deformation. Since the assumption of Lemma 5.2 holds in this case, we conclude immediately that:

THEOREM 5.3. If two graphs $G$ and $G^{\prime}$ can be transformed into each other by a finite sequence of admissible deformations, then $f(G)=f\left(G^{\prime}\right)$.

Both arrangement of blocks and turning around two vertices are admissible deformations for $|U|=2$. However, when $U$ contains three or more vertices, a bijection $\sigma: U \rightarrow U$ does not always yield an admissible deformation of $G=K \cup H$. The condition $(*)$ for the triplet $(H, U, \sigma)$ seems to be strong. For example, Tutte [2] has found certain classes of graphs $R_{3}, R_{4}, R_{5}$ as $H$, called rotors of order $3,4,5$ (Figure 6), which satisfy the condition $(*)$, but he failed to generalize them to ones of order 6 or more.

Each rotor $H$ has a cyclic symmetry, that is, there is an automorphism $\rho: H \rightarrow H$ which shifts cyclically each $u_{i} \in U$ to $u_{i+1} \in U$. Let $\sigma: U \rightarrow U$ be any bijection naturally induced by a reflection of the plane in an axis. Then $\sigma$ does not extend to an automorphism of the rotor $H$. It is easy to see that this triplet $(H, U, \sigma)$ satisfy the condition $(*)$ if $|U| \leq 5$. Note that the admissible deformations with rotors of order $3,4,5$ transform a graph $G$ into ones which are not 2-isomorphic to $G$.

When $|U| \leq 5$, every partition $\gamma_{i}$ of $U$ is reflective in some axis and the rotation $\rho^{k}: H \rightarrow H$ which carries the original axis to it induces an isomorphism between $H / \gamma_{i}$ and $H / \sigma\left(\gamma_{i}\right)$. But there is an unreflective partition $\gamma_{j}$ of $U$ if $|U| \geq 6$, say $\left(u_{1} ; u_{2}, u_{3} ; u_{4}, u_{5}, u_{6}\right)$ and no rotation $\rho^{k}$ induces an isomorphism between $H / \gamma_{j}$ and $H / \sigma\left(\gamma_{j}\right)$. That is why a rotor of order $\geq 6$ could not be constructed.

If a 2-isomorphic deformation, arrangement of blocks or turning around two vertices, is applicable to $G$, then $G$ has a cut vertex or a cut of two vertices, so $G$ is not 3-connected. Conversely if $G$ is 3-connected then there is no graph, except $G$ itself, which is 2 -isomorphic to $G$. Thus we cannot construct a 3 -connected graph which has the same polynomial as $G$ and which is 2-isomorphic to $G$ but not isomorphic to $G$. 
However, we can get pairs $\left(G, G^{\prime}\right)$ of 3,4 and 5-connected graphs which have the same polynomials, using rotors of order 3,4 and 5 , respectively; use the join $R_{k}+K_{m}$ of a rotor $R_{k}$ and a suitable complete graph $K_{m}$ as $H$, instead of $R_{k}$ $(k=3,4,5)$, to increase the connectivities of $G$ and $G^{\prime}$.

The failure in construction of such a pair of 6-connected graphs leads us naturally to the question whether or not the polynomial $f(G)$ is a complete invariant for 6connected graphs. As another question, we would like to ask whether or not the converse of Theorem 5.3 holds. Find other types of admissible deformations if it is not true.

Finally, we shall discuss the difference between the 2-isomorphism and the property of having the same polynomial. By Theorem 1.4, for two graphs $G$ and $G^{\prime}$, $f(G)=f\left(G^{\prime}\right)$ if and only if there is a bijection $\Theta: 2^{E(G)} \rightarrow 2^{E\left(G^{\prime}\right)}$ between the power sets of $E(G)$ and $E\left(G^{\prime}\right)$ such that $\omega(G-Y)=\omega\left(G^{\prime}-\Theta(Y)\right)$ for any subset $Y \subset E(G)$. This condition is equivalent to $\beta(G-X)=\beta\left(G^{\prime}-\Theta(X)\right)$ for any subset $X \subset E(G)$ and $\omega(G)=\omega\left(G^{\prime}\right)$, by Theorem 3.2.

Suppose that $\Theta: 2^{E(G)} \rightarrow 2^{E\left(G^{\prime}\right)}$ is compatible with inclusion, that is, $\Theta(A) \subset$ $\Theta(B) \subset E\left(G^{\prime}\right)$ if $A \subset B \subset E(G)$. Then there is a bijection $\theta: E(G) \rightarrow E\left(G^{\prime}\right)$ such that $\Theta(A)=\theta(A)$ for $A \subset E(G)$.

Let $A \subset E(G)$ be a cycle of $G$, precisely the edge set of a cycle and set $X=$ $E(G)-A$. The graph $G-X$ consists of only one cycle $A$ and several isolated vertices and hence $\beta(G-X)=\beta\left(G^{\prime}-\Theta(X)\right)=1$. Furthermore, $\beta(G-X \cup\{e\})=$ $\beta(G-\Theta(X \cup\{e\}))=0$ for any edge $e \in A$. This implies that also $\Theta(A)$ is a cycle of $G^{\prime}$, so $\Theta$ (or $\theta$ ) induces a one-to-one correspondence between cycles of $G$ and of $G^{\prime}$. By Whitney's result [5] previously mentioned, we conclude that $G$ and $G^{\prime}$ are 2 -isomorphic. Conversely, if $G$ and $G^{\prime}$ are 2-isomorphic, then there is obviously the $\operatorname{map} \theta: E(G) \rightarrow E\left(G^{\prime}\right)$ as above.

Now we shall describe this phenomenon in terms of resolutions. For two 2isomorphic graphs $G$ and $G^{\prime}$, we can construct two standard total resolutions for them in parallel and we can define a bijection $\theta: E(G) \rightarrow E\left(G^{\prime}\right)$, according to these. On the other hand, it will be observed that a standard total resolution of a graph $G=K \cup H$ with $H$ a rotor can be translated into a total resolution of $G^{\prime}=K \cup_{\sigma} H$ but it is not standard. We should understand how we can break the standardness of resolutions, preserving the polynomial $f(G)$. This might give us a hint to answer our previous questions.

\section{REFERENCES}

1. G. D. Birkhoff and D. Lewis, Chromatic polynomials, Trans. Amer. Math. Soc. 60 (1946), 355-451.

2. W. T. Tutte, Codichromatic graphs, J. Combin. Theory Ser. B 16 (1974), 168-174.

3. __ Graph theory, Encyclopedia of Mathematics, vol. 21, Addison-Wesley, 1984.

4. H. Whitney, Non-separable and planar graphs, Trans. Amer. Math. Soc. 34 (1932), 339362 .

5. __ 2-isomorphic graphs, Amer. J. Math. 55 (1933), 245-254.

Department of Information SCiEnCE, TOKyo Institute of Technology, OHOKAYAMA, MEGURO-KU, TOKYO 152 JAPAN 